\title{
Estimated pulmonary capillary wedge pressure assessed by speckle tracking echocardiography predicts successful ablation in paroxysmal atrial fibrillation
}

Masanori Kawasaki ${ }^{1 *}$, Ryuhei Tanaka², Taiji Miyake ${ }^{3}$, Reiko Matsuoka ${ }^{2}$, Mayumi Kaneda $^{2}$, Shingo Minatoguchi ${ }^{1}$, Takeshi Hirose ${ }^{2}$, Koji Ono ${ }^{2}$, Maki Nagaya² ${ }^{2}$ Hidemaro Sato ${ }^{4}$, Yoshiaki Kawase ${ }^{3}$, Shinji Tomita ${ }^{3}$, Kunihiko Tsuchiya ${ }^{3}$, Hitoshi Matsuo ${ }^{3}$, Toshiyuki Noda ${ }^{2}$ and Shinya Minatoguchi ${ }^{1}$

\begin{abstract}
Background: Atrial fibrillation (AF) is associated with left atrial (LA) remodeling caused by pressure and/or volume (LAV) overload. Increased pulmonary capillary wedge pressure (PCWP) represents LA pressure overload. We recently reported that pulmonary capillary wedge pressure (ePCWP) can be estimated by the kinetics-tracking (KT) index that combines LA function and volume using speckle tracking echocardiography (STE), and has a strong correlation with PCWP measured by right heart catheterization $(r=0.92)$. Therefore, we hypothesized that ePCWP is the best echocardiographic predictor of successful AF ablation.

Methods: We enrolled 137 patients with paroxysmal AF (age: $61 \pm 10$ years) who underwent pulmonary vein isolation. We measured LAV index, LA emptying function (EF) and LA stiffness during sinus rhythm before ablation using STE. PCWP was noninvasively estimated by STE as we previously reported. Parameters were compared between a group with AF recurrence ( $n=30$, age: $59 \pm 11$ years) and a group with successful ablation (sinus rhythm maintained for $>1$ year) ( $n=107$, age $61 \pm 11$ years).

Results: The ePCWP was correlated with PCWP measured by right heart catheterization $(r=0.76, p<0.01)$. Compared with the non-recurrence group ( $n=107$, age: $61 \pm 11$ ), the AF recurrence group had significantly increased ePCWP $(10.6 \pm 3.5$ vs $14.6 \pm 2.9 \mathrm{mmHg}, p<0.01)$, minimum LAV index $\left(29 \pm 12 \mathrm{ml} / \mathrm{m}^{2} \mathrm{vs} 37 \pm 14 \mathrm{ml} / \mathrm{m}^{2}\right.$, $p<0.01)$ and LA stiffness $(0.47 \pm 0.33$ vs $0.83 \pm 0.59, p<0.01)$, but lower total LA EF $(44 \pm 11 \%$ vs $39 \pm 13 \%, p<0.01)$ before ablation. In multivariate logistic regression analysis, ePCWP was the most significant independent predictor of successful ablation. Using $13 \mathrm{mmHg}$ of PCWP as the optimal cutoff value, the sensitivity and specificity for successful ablation were 73 and $77 \%$ (area under the curve $=0.81$ ), respectively.
\end{abstract}

Conclusion: The EPCWP that is measured by the combination of LA function and volume before ablation was a better predictor of the successful ablation compared with LA function and volume separately. The ePCWP estimated by STE is useful to predict the successful ablation in paroxysmal AF, and could be useful to improve candidate selection for AF ablation.

Keywords: Atrial fibrillation ablation, Recurrence, Pulmonary capillary wedge pressure, Speckle tracking echocardiography

\footnotetext{
* Correspondence: masanori@ya2.so-net.ne.jp

'Department of Cardiology, Gifu University Graduate School of Medicine, 1-1

Yanagido, Gifu 501-1194, Japan

Full list of author information is available at the end of the article
} 


\section{Background}

Atrial fibrillation (AF) is the most common sustained arrhythmia and is associated with left atrial (LA) enlargement, remodeling and fibrosis caused by LA pressure and/or volume (LAV) overload [1-3]. AF ablation is an important therapeutic modality because AF ablation has become an effective treatment strategy for patients with drug-refractory AF and is more effective than anti-arrhythmic medications $[4,5]$.

Many factors such as type of AF, LA size, hypertension, diabetes mellitus, renal function, age, CHADS2, R2CHADS2 or CHA2DS2-VASc score have been proposed as predictors of outcome after AF ablation [6-8]. Recently, left atrial appendage wall-motion velocity and sinus rhythm restoration and arrhythmia noninducibility have been proposed as predictors of outcome after AF ablation $[9,10]$. Although echocardiographic parameters have not been fully examined as predictors of ablation outcome except for LA dimension, volume and function [10-15], the ability of LA pressure to predict the success AF ablation is unknown.

We recently reported that estimated pulmonary capillary wedge pressure (ePCWP) has a strong correlation with PCWP measured by right heart catheterization $(r=0.92)$ [16, 17]. ePCWP is estimated by the kineticstracking (KT) index that is obtained by the combination of LA function and volume using speckle tracking echocardiography (STE). Therefore, we assessed the hypothesis that ePCWP as well as LA size may be useful predictors for successful AF ablation in patients with paroxysmal AF.

\section{Methods}

\section{Subjects and study protocol}

We enrolled 150 consecutive patients with paroxysmal AF undergoing pulmonary vein isolation (PVI) from June 2012 until January 2014. Paroxysmal AF was defined as self-terminating within 7 days after onset documented by routine electrocardiograms (ECG) or Holter ECG. Patients who met any of following criteria were excluded: an inadequate echo image to analyze LA function and volume, AF when echocardiography was performed, severe mitral regurgitation, or moderate or severe mitral stenosis. Patients who needed evaluation for heart failure underwent right heart catheterization including PCWP measurement. We validated the correlation between ePCWP and PCWP in these patients during sinus rhythm. All patients who underwent PVI were followed at the out-patient clinic of our hospital every month for at least 12 months after the procedure: an ECG was obtained every month and additional Holter recordings were obtained when the patient's symptoms suggested of AF. Recurrence of AF was defined as any episode of AF lasting $>30 \mathrm{~s}$ after AF ablation and was documented by ECG or Holter ECG. Transthoracic echocardiography was performed in the left lateral decubitus position by two experienced sonographers just before PVI (within two hours). Right heart catheterization was performed in the supine position without sedatives. The present study was approved by the institutional review board of Gifu Heart Center and all patients gave written informed consent before participation. The present study conforms to the principles outlined in the Declaration of Helsinki.

\section{Radiofrequency catheter ablation}

AF ablation was performed using an approach well documented by Eitel et al. [18]. Patients presenting $\mathrm{AF}$ at the beginning of the procedure were electrically cardioverted and ablation was performed during sinus rhythm. Circumferential LA ablation lines were placed around the antrum of the ipsilateral pulmonary veins. An irrigated radiofrequency ablation catheter was used to deliver 25-35 W of power. Voltage and pace mapping along the ablation line were used to identify and close conduction gaps after circumferential line placement. The electrical isolation of all pulmonary veins with bidirectional block was verified with a multipolar circular mapping catheter and was defined as the procedural endpoint.

\section{LA size, function and strain assessed by 2D-STE}

A standard echo-Doppler and two dimensional (2D)-STE examination were performed using a SC2000 ultrasound system (Siemens Medical Solutions Inc., Mountain View, CA, USA) with a 4V1c transducer (1.25-4.5 MHz). Echocardiographic measurements were made according to the American Society of Echocardiography criteria [19]. We examined the following parameters during sinus rhythm before PVI in apical 4-chamber view using offline software (Aquson, Sequoia, Siemens Medical Solutions Inc., Mountain View, CA, USA): maximum, pre-atrial contraction and minimum LAV; LA total and active emptying function (EF); longitudinal LA peak strain; and strain rate (SR) during systole and atrial contraction (Fig. 1). LAV, LA EF, longitudinal LA peak strain and SR were obtained from the timeLA volume curve and strain curve as we previously reported [2, 3, 16, 17]. LAV was indexed to body surface area. The reliability and the reproducibility of the STE method for quantification of LA function and volume were established in our previous study [2, 3, 16, 17]. Echocardiographic parameters were compared between the successful group (sinus rhythm was maintained for $>1$ year) and the recurrence group. The total and active LA EF were calculated as (maximum $\mathrm{LAV}$ - minimum $\mathrm{LAV}$ )/maximum LAV $\times 100 \%$, and (pre-atrial contraction LAV - minimum LAV)/pre-atrial contraction LAV $\times 100 \%$, respectively. 


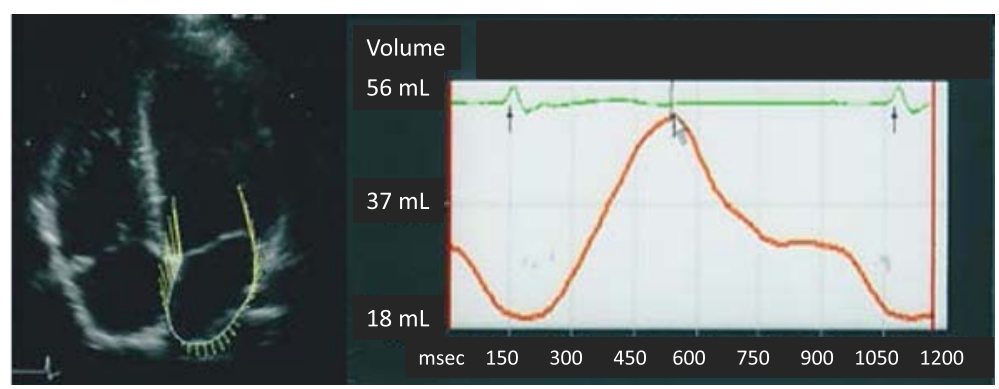

Fig. 1 Representative image of time-left atrial volume curve. (Left) velocity vector imaging of the left atrium from the apical 4-chamber view; (Right) orange line: time-left atrial volume curve; green line: electrocardiogram. LA: left atrium

\section{Pulmonary capillary wedge pressure and LA stiffness estimated by 2D-STE}

The combined assessment of LAV and LA function would be useful to estimate pre-atrial contraction LV filling pressure, because LAV and LA function are directly influenced by LV diastolic function. The KT index is calculated using the combination of LA function and volume. As LV diastolic function continues to decrease, LA volume continues to increase [20, 21]. Therefore, we employed LA volume as the denominator in the KT index to evaluate PCWP. Moreover, Hsiao et al. previously reported the logarithmic correlation between LV filling pressure and LA distensibility [( $\max$ LAVI - min LAVI)/min LAVI] that is similar to the total LAEF [(max LAVI - min LAVI)/max LAVI] [21]. Therefore, the $\mathrm{KT}$ index is defined as $\log _{10}$ (active LA EF/minimum LAV index) as we previously reported $[16,17]$. Multivariate regression analysis showed that the ePCWP is calculated as $10.8-12.4 \times \mathrm{KT}$ index $[16,17]$. The reliability of ePCWP has been well-established in several previous studies $[16,17,22]$. The ePCWP measured by this function just before right heart catheterization (within one hour) was strongly correlated with PCWP obtained by right heart catheterization $(r=0.92)$ in patients with normal sinus rhythm [16]. The intraobserver correlation coefficient and variability in ePCWP were 0.99 and $0.9 \pm 1.5 \%$, respectively. The interobserver correlation coefficient and variability in ePCWP were 0.99 and $2.5 \pm 1.8 \%$, respectively [16]. Moreover, we estimated LA stiffness using ePCWP as ePCWP/LA strain obtained by STE [23, 24].

\section{Statistical analyses}

Continuous variables are expressed as the mean \pm standard deviation, and categorical variables are presented as frequency and percentage. Differences in the categorical variables between the groups were tested by a Chi-square test or Fisher's exact test, and differences in the continuous variables between the groups were tested by an unpaired $t$-test. The differences in CHA2DS2-VASc score between groups were tested by a Mann-Whitney $U$-test. A multivariate logistic regression analysis was performed using data from both groups to determine the independent echocardiographic predictors of successful AF ablation. Only parameters that were significant in univariate analysis were used in the multivariate regression model. Receiver operating characteristic (ROC) curve analysis was used to discriminate between the recurrence and successful groups using the various echo parameters, and we determined the sensitivity, specificity, positive and negative predictive values and area under the curve (AUC) for each parameter. Differences in AUC among the echo parameters were tested by the method established by Hanley and McNeil [25]. A p value $<0.05$ was considered significant. Statistical analyses were performed using Stat View version 5.0 (SAS Institution Inc., Cary, NC, USA).

\section{Results}

Of 150 consecutive patients, 10 patients were excluded because of inadequate echo images of the LA $(n=6)$ and inability to perform echocardiography during sinus rhythm $(n=4)$. We excluded patients with severe mitral regurgitation $(n=2)$ and moderate or severe mitral stenosis $(n=1)$. Accordingly, we enrolled 137 patients (61 \pm 10 years) who had echocardiography during sinus rhythm and underwent PVI for paroxysmal AF. Of 137 patients, 109 patients underwent their first AF ablation procedure and 28 patients underwent their second AF ablation procedure. Of 137 patients, 57 patients underwent right heart catheterization including PCWP measurement.

The baseline characteristics of the study population are listed in Table 1. Echocardiographic parameters of patients with successful ablation and patients with AF recurrence are listed in Table 2 . Thirty patients had AF recurrence ( $22 \%$ of all patients) and the mean time from ablation to AF was $5 \pm 2$ months: all AF episodes were confirmed by ECG or Holter ECG. There was a strong correlation between PCWP obtained by right heart catheterization and ePCWP $(r=0.76, p<0.001)$ in 57 patients (Fig. 2). The ePCWP, LA stiffness, maximum and 
Table 1 Baseline characteristics of the study population

\begin{tabular}{|c|c|c|c|}
\hline & $\begin{array}{l}\text { Recurrence Group } \\
(n=30)\end{array}$ & $\begin{array}{l}\text { Successful Group } \\
(n=107)\end{array}$ & $p$ value \\
\hline Age, years & $59.4 \pm 10.5$ & $61.3 \pm 10.7$ & 0.22 \\
\hline Females, n (\%) & $9(30)$ & $31(29)$ & 0.91 \\
\hline Body mass index, $\mathrm{kg} / \mathrm{cm}^{2}$ & $24.2 \pm 3.3$ & $23.9 \pm 3.2$ & 0.43 \\
\hline Heart rate, beat per minute & $72 \pm 15$ & $73 \pm 15$ & 0.77 \\
\hline Systolic blood pressure, mmHg & $121 \pm 17$ & $121 \pm 15$ & 0.83 \\
\hline Diastolic blood pressure, $\mathrm{mmHg}$ & $75 \pm 14$ & $73 \pm 11$ & 0.45 \\
\hline $\mathrm{eGFR}, \mathrm{ml} / \mathrm{min} / 1.73 \mathrm{~m}^{2}$ & $73.8 \pm 16.2$ & $72.8 \pm 17.5$ & 0.82 \\
\hline Previous ablation, n (\%) & $9(30)$ & $19(18)$ & 0.14 \\
\hline Hypertension, n (\%) & $14(47)$ & $49(46)$ & 0.93 \\
\hline Diabetes mellitus, n (\%) & $5(17)$ & $17(16)$ & $>0.99$ \\
\hline Coronary artery disease, n (\%) & $2(7)$ & $6(6)$ & $>0.99$ \\
\hline Smoking, n (\%) & $5(17)$ & $17(16)$ & $>0.99$ \\
\hline Anti-arrhythmic drugs, n (\%) & $16(53)$ & $49(46)$ & 0.47 \\
\hline Congestive heart failure, n (\%) & $3(10)$ & $9(8)$ & 0.73 \\
\hline CHA2DS2-VASc score & $1.4 \pm 1.3$ & $1.4 \pm 1.2$ & 0.83 \\
\hline
\end{tabular}

$A F$ atrial fibrillation, eGFR estimated glomerular filtration rate

minimum LAV index and the ratio of peak early transmitral flow velocity (E) to the regional tissue velocity of the mitral annulus measured during early filling $\left(E / e^{\prime}\right)$ before $\mathrm{AF}$ ablation in the recurrence group were increased compared with the successful group (Table 2). The total and active LA EF and longitudinal LA peak strain were decreased before AF ablation in the recurrence group. However, there was no significant difference in left ventricular ejection fraction or LA dimension between the two groups.
Echocardiographic predictors of rhythm outcomes after AF ablation were evaluated by a multivariate logistic regression analysis (Table 3 ). All variables with a $p$ value $<0.05$ in univariate testing were included in multivariate logistic regression analysis. However, maximum LAV index, active LAV index and total LA EF could not be included in the multivariate analysis due to multicollinearity. In multivariate analysis, ePCWP assessed during sinus rhythm before $\mathrm{AF}$ ablation had the most significant association with the outcome of AF ablation.

Table 2 Comparison of echo parameters before AF ablation between in groups with successful and non-successful outcomes

\begin{tabular}{llll}
\hline & $\begin{array}{l}\text { Recurrence Group } \\
(n=30)\end{array}$ & $\begin{array}{l}\text { Successful Group } \\
(n=107)\end{array}$ & $p$ value \\
\hline LV EF, \% & $63 \pm 8$ & $64 \pm 8$ & 0.74 \\
E/e' & $12 \pm 4$ & $10 \pm 3$ & 0.039 \\
LAD, mm & $43 \pm 5$ & $41 \pm 6$ & 0.16 \\
LV mass index, g/m ${ }^{2}$ & $97 \pm 17$ & $94 \pm 17$ & 0.41 \\
Max LAVI, $\mathrm{mL} / \mathrm{m}^{2}$ & $58 \pm 15$ & $49 \pm 15$ & 0.005 \\
Min LAVI, $\mathrm{mL} / \mathrm{m}^{2}$ & $37 \pm 14$ & $29 \pm 12$ & 0.002 \\
Total LAEF, \% & $39 \pm 13$ & $44 \pm 11$ & 0.030 \\
Active LAEF, \% & $20 \pm 7$ & $25 \pm 10$ & 0.033 \\
Longitudinal LA peak strain & $23 \pm 9$ & $28 \pm 13$ & 0.031 \\
LA SR during systole, s ${ }^{-1}$ & $0.95 \pm 0.42$ & $1.12 \pm 0.52$ & 0.11 \\
LA SR during AC, s ${ }^{-1}$ & $-0.84 \pm 0.44$ & $-1.01 \pm 0.62$ & 0.15 \\
ePCWP, mmHg & $14.6 \pm 2.9$ & $10.6 \pm 3.5$ & $<0.001$ \\
LA stiffness & $0.83 \pm 0.59$ & $0.47 \pm 0.33$ & $<0.001$ \\
\hline AF & & \\
\hline
\end{tabular}

$A F$ atrial fibrillation, $L V E F$ left ventricular ejection fraction, E/e' ratio of early filling velocity to myocardial tissue velocity, $L A D$ left atrial dimension, $M a x$ LAVI maximum left atrial volume index, Min LAVI minimum left atrial volume index, Total LAEF left atrial total emptying fraction, Active LAEF left atrial active emptying function, $L A S R$ left atrial strain rate, $A C$ atrial contraction, $e P C W P$ estimated pulmonary capillary wedge pressure 


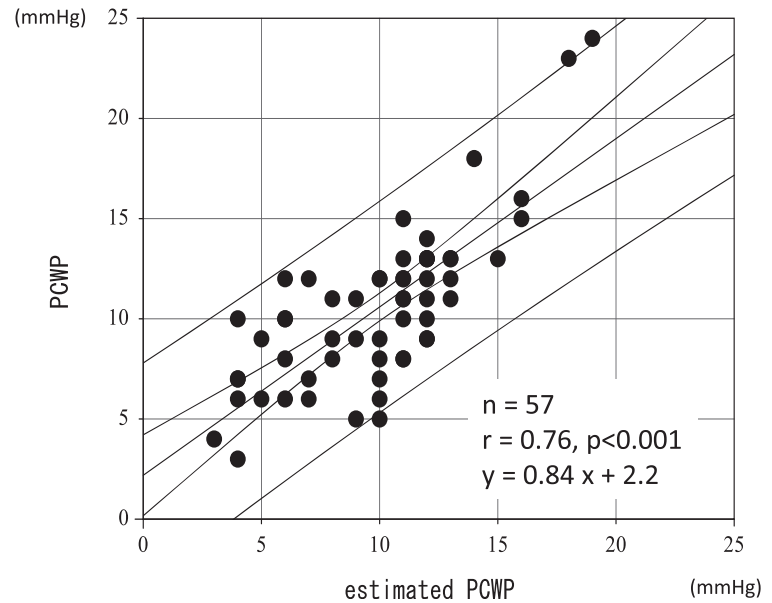

Fig. 2 Relationship between pulmonary capillary wedge pressure measured by right heart catheterization and speckle tracking echocardiography. Center line indicates the regression line. Inner lines indicate the $95 \%$ confidence intervals of the regression line. Outer lines indicate the $95 \%$ confidence intervals of the raw data. PCWP: pulmonary capillary wedge pressure
Using $13 \mathrm{mmHg}$ of ePCWP as the optimal cutoff value, the sensitivity and specificity for successful ablation were 73 and $77 \%$, respectively, and the positive and negative predictive values were 92 and $44 \%$, respectively. The AUC for ePCWP (0.81) was better than that for LAD $(0.58, p<0.001)$, total LA EF $(0.64, p=0.022)$, active LA $\mathrm{EF}(0.63, p=0.010)$ and $\mathrm{E} / \mathrm{e}^{\prime}(0.60, p=0.044)$ (Fig. 3, Table 4). There was a tendency for the AUC for ePCWP to be better than that for minimum LAVI $(0.68, p=0.056)$. All patients with ePCWP $<10 \mathrm{mmHg}$ before AF ablation regardless of LA dimension had successful AF ablation (Fig. 4). Therefore, we also employed $10 \mathrm{mmHg}$ as a second cutoff value for ePCWP to increase the positive predictive value rather than to maintain a high sensitivity. Using $10 \mathrm{mmHg}$ of ePCWP as a second cutoff value, the sensitivity and specificity for successful ablation were 32 and $100 \%$, respectively, and the positive and negative predictive values were 100 and $29 \%$, respectively. Predictive accuracies of different ePCWP to predict successful AF ablation were shown in Table 5.

\section{Discussion}

The present study demonstrated that ePCWP estimated by the KT index was most useful predictor among echo parameters for successful AF ablation in patients with

Table 3 Multivariate logistic regression analysis to determine the independent predictors of a successful AF ablation and subanalysis on first and second AF ablation

\begin{tabular}{|c|c|c|c|c|}
\hline \multicolumn{5}{|l|}{ All patients $(n=137)$} \\
\hline & $p$ value & Odds ratio & $95 \%$ confidence interval & \\
\hline Minimum LAVI & 0.011 & 1.093 & 1.021 & 1.171 \\
\hline ePCWP & $<0.001$ & 0.482 & 0.340 & 0.682 \\
\hline Longitudinal LA peak strain & 0.072 & 0.940 & 0.879 & 1.005 \\
\hline$E / e^{\prime}$ & 0.56 & 1.041 & 0.908 & 1.192 \\
\hline LA stiffness & 0.19 & 0.296 & 0.047 & 1.851 \\
\hline \multicolumn{5}{|l|}{ First AF ablation $(n=109)$} \\
\hline & $p$ value & Odds ratio & $95 \%$ confidence interval & \\
\hline Minimum LAVI & 0.029 & 1.092 & 1.009 & 1.182 \\
\hline ePCWP & $<0.001$ & 0.419 & 0.257 & 0.682 \\
\hline Longitudinal LA peak strain & 0.094 & 0.930 & 0.855 & 1.012 \\
\hline$E / e^{\prime}$ & 0.63 & 1.038 & 0.889 & 1.214 \\
\hline LA stiffness & 0.39 & 0.377 & 0.041 & 3.462 \\
\hline \multicolumn{5}{|l|}{ Second AF ablation $(n=28)$} \\
\hline & $p$ value & Odds ratio & $95 \%$ confidence interval & \\
\hline Minimum LAVI & 0.15 & 1.113 & 0.959 & 1.322 \\
\hline ePCWP & 0.057 & 0.579 & 0.329 & 1.018 \\
\hline Longitudinal LA peak strain & 0.97 & 0.967 & 0.845 & 1.107 \\
\hline$E / e^{\prime}$ & 0.52 & 1.110 & 0.809 & 1.521 \\
\hline LA stiffness & 0.30 & 0.122 & 0.002 & 6.697 \\
\hline
\end{tabular}

$A F$ atrial fibrillation, $e P C W P$ estimated pulmonary capillary wedge pressure, E/e' ratio of early filling velocity to myocardial tissue velocity, $L A$ left atrial 

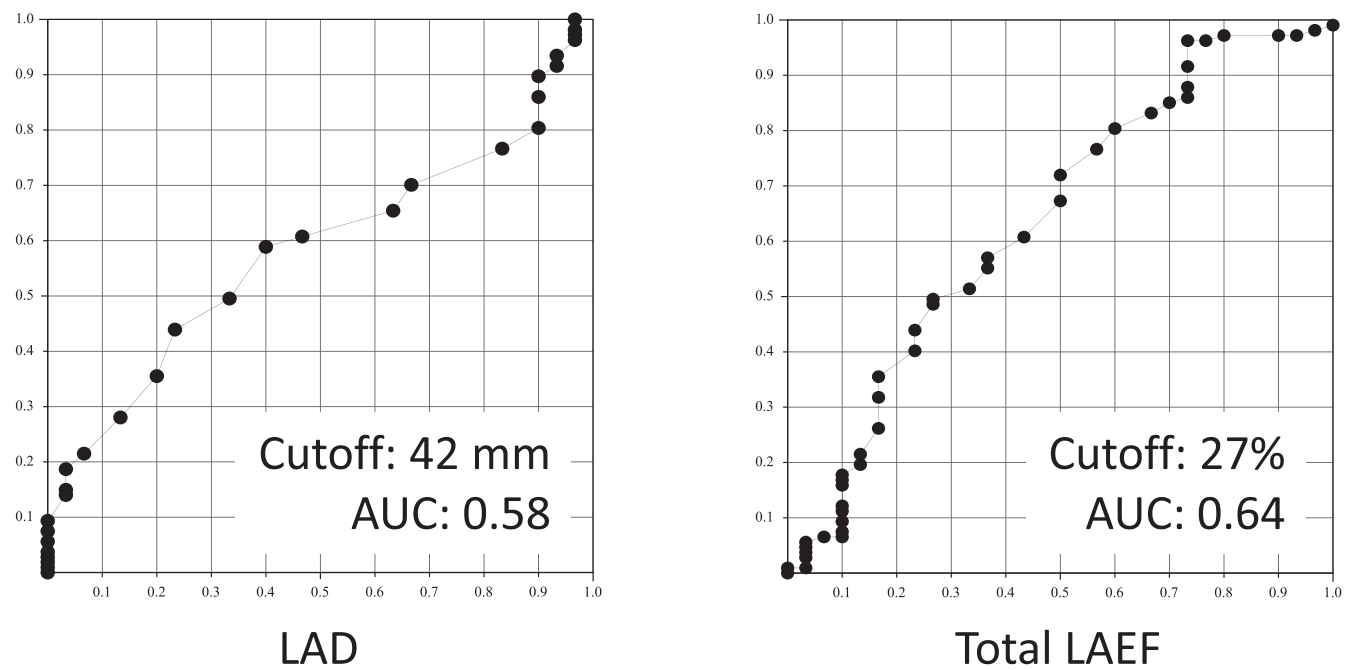

Total LAEF

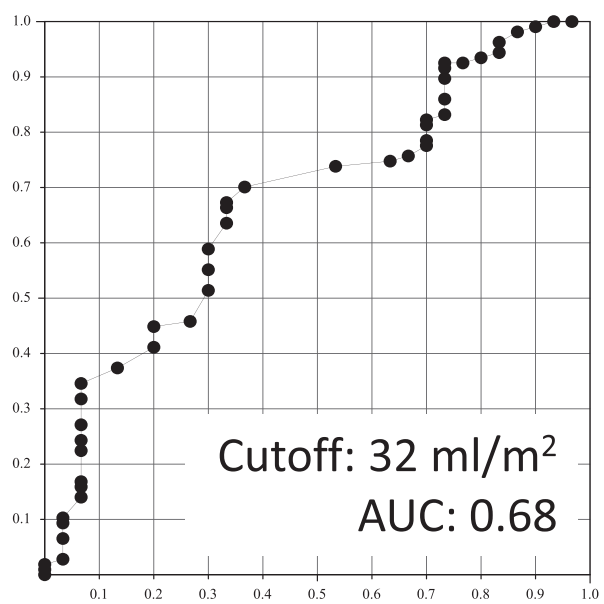

Minimum LAVI

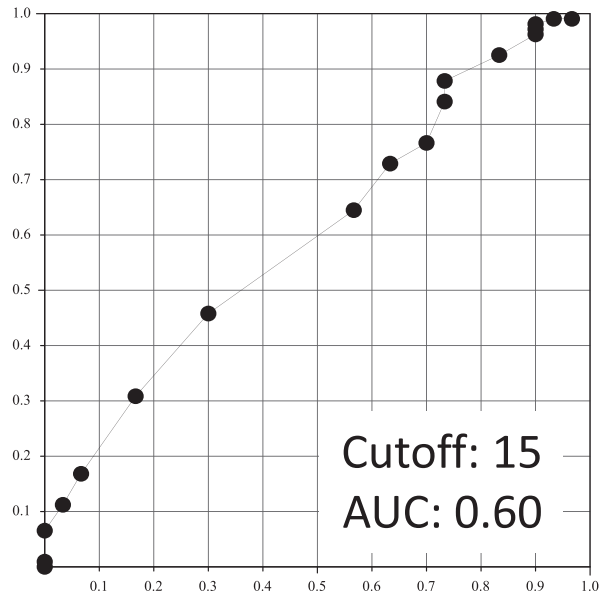

$E / e^{\prime}$

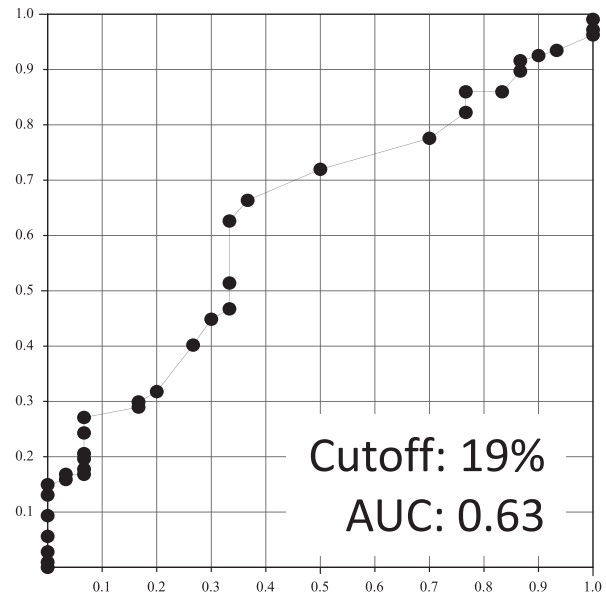

Active LAEF

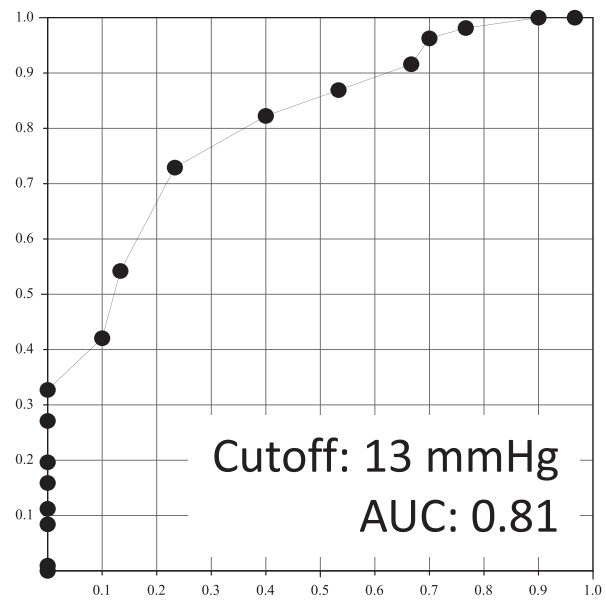

Estimated PCWP

Fig. 3 Receiver operating characteristic curve analyses of echo parameters for predicting successful AF ablation. LAD: left atrial dimension; AUC: area under the curve; Min LAVI: minimum left atrial volume index; Max LAVI: maximum left atrial volume index; LAEF: left atrial emptying fraction; PCWP: pulmonary capillary wedge pressure; E/e': ratio of early filling velocity to myocardial tissue velocity 
Table 4 Sensitivity and specificity of echo parameters to predict successful AF ablation

\begin{tabular}{|c|c|c|c|c|c|c|}
\hline & Cut off & Sensitivity \% & Specificity \% & PPV \% & NPV \% & AUC (95\% Cl) \\
\hline $\mathrm{LAD}, \mathrm{mm}$ & 42 & 60 & 59 & 29 & 84 & $0.58(0.50-0.66)$ \\
\hline Min LAVI, mL & 32 & 67 & 66 & 36 & 88 & $0.68(0.60-0.76)$ \\
\hline Max LAVI, mL & 51 & 70 & 60 & 33 & 88 & $0.66(0.58-0.74)$ \\
\hline Total LAEF, \% & 27 & 27 & 96 & 67 & 82 & $0.64(0.56-0.72)$ \\
\hline Active LAEF, \% & 19 & 63 & 66 & 35 & 87 & $0.63(0.55-0.71)$ \\
\hline ePCWP, mmHg & 13 & 77 & 73 & 44 & 92 & $0.81(0.74-0.88)$ \\
\hline
\end{tabular}

$A F$ atrial fibrillation, $P P V$ positive predictive value, NPV negative predictive value, $A U C$ area under the curve, $L A D$ left atrial dimension, Min $L A V I$ minimum left atrial volume index, Max LAVI maximum left atrial volume index, LAEF left atrial emptying fraction, ePCWP estimated pulmonary capillary wedge pressure, $\mathrm{Cl}$ confidence interval

paroxysmal AF. The association between AF ablation outcome and $\mathrm{ePCWP}$, calculated by the combination of LA function and volume, was stronger than the association observed using LA function and volume separately.

\section{AF ablation outcome and echocardiographic parameters} Recently, there were some studies that reported that LA function assessed by STE instead of LA size is a useful predictor for the outcome of AF ablation. Morris et al. reported that LA diastolic function assessed by LA strain (AUC: 0.827) and LA systolic function assessed by SR during atrial contraction (AUC: 0.806) in patients with paroxysmal AF and low CHADS2 score were useful predictors of AF ablation outcome [26]. Tops et al. reported that LA strain predicts reverse remodeling after AF ablation [27]. In the present study, LA strain was lower in the recurrence group than in

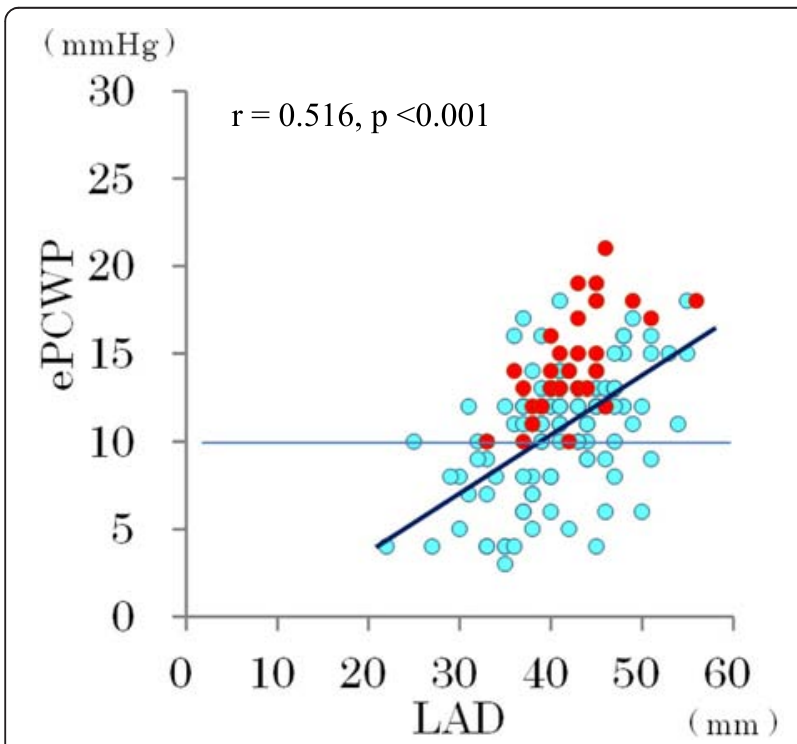

Fig. 4 Left atrial diameter and pulmonary capillary wedge pressure as predictors of successful AF ablation. ePCWP: estimated pulmonary capillary wedge pressure; LAD: left atrial dimension; blue circle: AF recurrence; red circle: successful AF ablation; blue broken line shows $10 \mathrm{mmHg}$ of ePCWP the successful group, and this result is consistent with the findings of Morris et al. Furthermore, Montserrat et al. reported that patients with successful AF ablation had better total LA EF than those with AF recurrence, and total LA EF was an independent predictor of arrhythmia elimination after the first AF ablation [28]. Taken together, LA function is more associated with the outcome of AF ablation than LA size. However, regarding LA pressure, there are very few STE studies that evaluated noninvasive predictors of the successful AF ablation. The present study evaluated the predictive value of LA pressure on the success of AF ablation.

\section{AF ablation outcome and LA size and function}

Among several echo parameters including estimated LA pressure and LA stiffness, we found that ePCWP is the most reliable echo parameter to predict AF ablation outcome. Using $13 \mathrm{mmHg}$ of ePCWP as an optimal cutoff value, the positive predictive value for successful AF ablation was $92 \%$ (95\% confidence interval: 87 - $97 \%$ ). Using $10 \mathrm{mmHg}$ of ePCWP as a second optimal cutoff value, the positive predictive value for successful AF ablation was $100 \%$. These results suggest that ePCWP measured by STE before the procedure may be useful to predict of the outcome one year after AF ablation.

The ePCWP can be calculated by the KT index that consists of the combination of LA function (active LA EF) and LA volume (minimum LAV index). This suggests that LA pressure may be a more important factor for the outcome of AF ablation than LA function or volume evaluated separately. This also suggests that ePCWP may be the useful predictor of the outcome of AF ablation with high reliability, since the AUC was 0.81 based on ROC curve analysis. On the other hand, the other echo parameters had lower AUCs (0.58 - 0.68) than ePCWP.

In our previous study for the development of ePCWP, STE was performed just before right heart catheterization (within one hour). However, in the present study, STE was performed one day before AF ablation. Therefore, the correlation coefficient between ePCWP and PCWP measured 
Table 5 Predictive accuracies of different estimated pulmonary capillary wedge pressures to predict successful AF ablation

\begin{tabular}{lllll}
\hline ePCWP & Sensitivity & Specificity & PPV & NPV \\
\hline $10 \mathrm{mmHg}$ & $32(24-40)$ & $100(100-100)$ & $100(100-100)$ & $29(21-37)$ \\
$11 \mathrm{mmHg}$ & $42(34-50)$ & $90(85-95)$ & $94(90-98)$ & $30(22-38)$ \\
$12 \mathrm{mmHg}$ & $54(46-62)$ & $87(81-93)$ & $93(89-97)$ & $34(26-42)$ \\
$13 \mathrm{mmHg}$ & $72(64-80)$ & $77(70-84)$ & $92(87-97)$ & $44(36-52)$ \\
$14 \mathrm{mmHg}$ & $82(76-88)$ & $60(52-68)$ & $88(83-93)$ & $49(41-57)$ \\
$15 \mathrm{mmHg}$ & $87(81-92)$ & $47(39-55)$ & $85(79-91)$ & $50(42-58)$ \\
$16 \mathrm{mmHg}$ & $92(87-97)$ & $34(26-42)$ & $83(77-89)$ & $53(45-61)$ \\
$17 \mathrm{mmHg}$ & $96(93-99)$ & $30(22-38)$ & $82(76-88)$ & $69(61-77)$ \\
$18 \mathrm{mmHg}$ & $98(96-100)$ & $24(17-31)$ & $80(73-87)$ & $77(70-84)$ \\
$19 \mathrm{mmHg}$ & $100(100-100)$ & $10(5-15)$ & $79(72-86)$ & $100(100-100)$
\end{tabular}

Data are percentages. Numbers in parentheses are $95 \%$ confidence intervals. AF atrial fibrillation, ePCWP estimated pulmonary capillary wedge pressure, PPV positive predictive value, NPV negative predictive value

Note that the positive predictive values are useful to estimate the probability of successful AF ablation

by right heart catheterization was lower $(r=0.76)$ than the previous training study $(r=0.92)[16]$.

\section{Differences between $\mathrm{E} / \mathrm{e}^{\prime}$ and $\mathrm{ePCWP}$}

$\mathrm{E} / \mathrm{e}^{\prime}$ has been proposed to estimate LV filling pressure [29-31]. However, E/e' does not reflect the condition of diastasis that may occur during mid and late diastole, or atrial contraction that is directly affected by LV stiffness. Therefore, E/e' is a parameter that evaluates only early diastole. To overcome the limitations of E/e', we proposed a combination of minimum LAVI and active LAEF (KT index) that evaluates LA features throughout diastole to estimate PCWP.

\section{AF ablation outcome and CHADS2 score}

Kornej et al. reported that R2CHADS2 score, CHA2DS2VASc score and LA dimension were significant predictors for late recurrence of AF after AF ablation. However, R2CHADS2 score and CHA2DS2-VASc score showed only low predictive value (AUC: 0.541 and 0.545 , respectively) [13]. The statistical significance of the predictive value of these parameters despite a low AUC was due to a large population in the study [13].

\section{Study limitations}

There are several limitations of this study. First, the study population was small, and only patients with paroxysmal AF were included. Therefore, there were no differences in clinical parameters between the two groups. Second, the rate of AF recurrence after AF ablation could have been underestimated. However, the protocol for detection of AF recurrence seems to be an ideal scenario from a practical point of view. Third, we focused only on echocardiographic parameters to predict the outcome of AF ablation. There is a possibility that other factors such as the duration of paroxysmal AF or the $\mathrm{P}$ wave duration may predict AF recurrence after ablation.
Further study in a larger population including patients with persistent AF will be needed.

\section{Conclusions}

An elevated ePCWP before AF ablation, but not an enlarged LA size, assessed by STE was the best predictor of $\mathrm{AF}$ recurrence after AF ablation, suggesting a strong relationship between LA pressure and the progression of LA remodeling responsible for $\mathrm{AF}$. The echocardiographic parameter of ePCWP assessed by the KT index could be useful to improve candidate selection for AF ablation.

\section{Abbreviations}

AF: atrial fibrillation; LA: left atrial; LAV: left atrial volume; PCWP: pulmonary capillary wedge pressure; ePCWP: estimated pulmonary capillary wedge pressure; $\mathrm{KT}$ index: kinetics-tracking index; STE: speckle tracking echocardiography; ECG: electrocardiogram; EF: emptying function; ROC: receiver operating characteristic; AUC: area under the curve.

\section{Competing interests}

The authors declare that they have no competing interests.

\section{Authors' contributions}

MK and RT analyzed data and wrote the manuscript. TM, RM, MK, SM, TH, $\mathrm{KO}, \mathrm{YK}, \mathrm{ST}$ and KT carried out AF ablation and analyzed data. NM and HS performed statistical and ultrasound analysis. HM, TN and SM revised the manuscript. All authors read and approved the final manuscript.

\section{Acknowledgements}

We thank Mr. Hirohito Nakata for his technical assistant of this manuscript.

\section{Author details}

${ }^{1}$ Department of Cardiology, Gifu University Graduate School of Medicine, 1-1 Yanagido, Gifu 501-1194, Japan. Department of Cardiology, Gifu Prefectural General Medical Center, Gifu, Japan. ${ }^{3}$ Department of Cardiology, Gifu Heart Center, Gifu, Japan. ${ }^{4}$ Department of Internal Medicine, Sawada Hospital, Gifu, Japan.

Received: 12 December 2015 Accepted: 19 January 2016 $\mathrm{BH}, \mathrm{H}, \mathrm{s}, \mathrm{s}$

\section{References}

1. Benjamin EJ, Wolf PA, D'Agostino RB, Silbershatz H, Kannel WB, Levy D. Impact of atrial fibrillation on the risk of death: the Framingham Heart Study. Circulation. 1998;98:946-52. 
2. Hirose T, Kawasaki M, Tanaka R, Ono K, Watanabe T, Iwama M, et al. Left atrial function assessed by speckle tracking echocardiography as a predictor of new-onset non-valvular atrial fibrillation: result from a prospective study in 580 adults. Eur Heart J Cardiovasc Imaging. 2012;13:243-50.

3. Kojima T, Kawasaki M, Tanaka R, Ono K, Hirose T, Iwama M, et al. Left atrial global and regional function in patients with paroxysmal atrial fibrillation has already been impaired before enlargement of left atrium: velocity vector imaging echocardiography study. Eur Heart J Cardiovasc Imaging. 2012;13:227-34

4. Cappato R, Calkins H, Chen SA, Davis W, lesaka Y, Kalman J, et al. Update worldwide survey on the method, efficacy, and safety of catheter ablation for human atrial fibrillation. Circ Arrhythm Electrophysiol. 2010;3:32-8.

5. Piccini JP, Lopes RD, Kong MH, Hasselblad V, Jackson K, Al-Khatib SM. Pulmonary vein isolation for the maintenance of sinus rhythm in patients with atrial fibrillation: a meta-analysis of randomized, controlled trials. Circ Arrhythm Electrophysiol. 2009;2:626-33.

6. Calkins H, Kuck KH, Cappato R, Brugada J, Camm AJ, Chen SA, et al. 2012 HRS/EHRA/ECAS expert consensus statement on catheter and surgical ablation of atrial fibrillation: recommendations for patient selection, procedural tevhniques, patient management and follow-up, definitions, endopoints, and research trial design. J Interv Card Electrophysiol. 2012;33: $171-257$.

7. $\quad$ ic LM, Goldenberg EM. CHADS 2 score predicts time interval free of atrial fibrillation in patients with symptomatic paroxysmal atrial fibrillation. Int J Cardiol. 2010;145:576-7.

8. Kornej J, Hindricks G, Kosiuk J, Arya A, Sommer P, Husser D, et al. Comparison of CHADS2, R2CHADS2 and CHA2DS2-VASC scores for the prediction of rhythm outcomes after catheter ablation of atrial fibrillation: the Leipzig heart center AF ablation registry. Circ Arrhythm Electrophysiol. 2014;7:281-7.

9. Ariyama M, Kato R, Matsumura M, Yoshimoto H, Nakajima Y, Nakano S, et al. Left atrial appendage wall-motion velocity associates with recurrence of nonparoxysmal atrial fibrillation after catheter ablation. Echocardiography. 2015;32:272-80

10. Fiala M, Bulková V, Škňouřil L, Nevřalová R, Toman O, Januška J, et al. Sinus rhythm restoration and arrhythmia noninducibility are major predictors of arrhythmia-free outcome after ablation for long-standing persistent atrial fibrillation: a prospective study. Heart Rhythm. 2015;12:687-98.

11. D'Ascenzo F, Crleto A, Biondi-Zoccai G, Anselmino M, Ferraris F, di Biase L, et al. Which are the most reliable predictors of recurrence of atrial fibrillation after transcatheter ablation? : a meta-analysis. Int J Cardiol. 2013; 167:1984-9.

12. Shin SH, Park MY, Oh WJ, Hong SJ, Pak HN, Song WH, et al. Left atrial volume is a predictor of atrial fibrillation recurrence after catheter ablation. J Am Soc Echocardiogr. 2008;21:697-702.

13. Zhuang J, Wang Y, Tang K, Li X, Peng W, Liang C, et al. Association between left atrial size and atrial fibrillation recurrence after single circumferential pulmonary vein isolation: a systematic review and meta-analysis of observational studies. Europace. 2012:14:638-45.

14. Nattel S, Burstein B, Dobrev D. Atrial remodelling and atrial fibrillation: mechanisms and implications. Circ Arrhythmia Electrophysiol. 2008;1:62-73.

15. Wakili R, Voigt N, Kaab S, Dobrev D, Nattel S. Recent advances in the molecular pathophysiology of atrial fibrillation. J Clin Invest. 2011;121:2955-68.

16. Kawasaki M, Tanaka R, Ono K, Minatoguchi S, Watanabe T, Iwama M, et al. A novel ultrasound predictor of pulmonary capillary wedge pressure assessed by the combination of left atrial volume and function: a speckle tracking echocardiography study. J Cardiol. 2015;66:253-62.

17. Kawase Y, Kawasaki M, Tanaka R, Nomura N, Fujii Y, Ogawa K, et al. Noninvasive estimation of pulmonary capillary wedge pressure in patients with mitral regurgitation: a speckle tracking echocardiography study. J Cardiol. 2016:67:192-8.

18. Eitel C, Hindricks G, Sommer P, Graspar T, Kircher S, Wetzel U, et al. Circumferential pulmonary vein isolation and liner left atrial ablation as a single-catheter technique to achieve bidirectional conduction block: the pace-and-ablate approach. Heart Rhythm. 2009;7:157-64

19. Lang RM, Bierig M, Devereux RB, Flachskampf FA, Foster E, Pellikka PA, et al. Recommendations for chamber quantification: a report from the American Society of Echocardiography's Guidelines and Standards Committee and the Chamber Quantification Writing Group, developed in conjunction with the European Association of Echocardiography, a branch of the European Society of Cardiology. J Am Soc Echocardiogr. 2005;18:1440-63.
20. Pritchett AM, Mahoney DW, Jacobsen SJ, Rodeheffer RJ, Karon BL, Redfield MM. Diastolic dysfunction and left atrial volume: a population-based study. J Am Coll Cardiol. 2005;45:87-92.

21. Hsiao SH, Huang WC, Lin KL, Chiou KR, Kuo FY, Lin SK, et al. Left atrial distensibility and left ventricular filling pressure in acute versus chronic severe mitral regurgitation. Am J Cardiol. 2010;105:709-15.

22. Kawasaki M, Tanaka R, Ono K, Minatoguchi S, Watanabe T, Arai M, Nishigaki K, Noda T, Watanabe S, Minitoguchi S. Impacts of gender and healthy aging on pulmonary capillary wedge pressure estimated by the kinetics-tracking index using two-dimensional speckle tracking echocardiography. Hyperten Res 2015 (in press)

23. Mirsky I. Assessment of diastolic function: suggested methods and future considerations. Circulation. 1984;69:836-41.

24. Mirsky I, Parmley WW. Assessment of passive elastic stiffness for isolated heart muscle and intact heart. Circ Res. 1973:33:233-43.

25. Hanley JA, MacNeil BJ. The method of comparing the area under receiver operating characteristics curves derived from the same cases. Radiology. 1983;148:839-43.

26. Morris DA, Parwani A, Huemer M, Wutzlier A, Bekfani T, Attanasio P, et al. Clinical significance of the assessment of the systolic and diastolic myocardial function of the left atrium in patients with paroxysmal atrial fibrillation and low CHADS2 index treated with catheter ablation therapy. Am J Cardiol. 2013;111:1002-11.

27. Tops LF, Delgado V, Bertini M, Marsan NA, Den Uijl DW, Trines SAIP, et al. Left atrial strain predicts reverse remodeling after catheter ablation for atrial fibrillation. J Am Coll Cardiol. 2011;57:324-31.

28. Montserrat S, Gabrielli L, Borras R, Poyatos S, Berruezo A, Bijnens B, et al. Left atrial size and function by three-dimensional echocardiography to predict arrhythmia recurrence after first and repeated ablation of atrial fibrillation. Eur Heart J Cardiovasc Imaging. 2014;15:515-22.

29. Nagueh SF, Middleton KJ, Kopelen HA, Zoghbi WA, Quinones MA. Doppler tissue imaging: a noninvasive technique for evaluation of left ventricular relaxation and estimation of filling pressures. J Am Coll Cardiology. 1997;30: 1527-33.

30. Nagueh SF, Mikati I, Kopelen HA, Middleton KJ, Quinones MA, Zoghbi WA Doppler estimation of left ventricular filling pressure in sinus tachycardia: a new application of tissue Doppler imaging. Circulation. 1998;98:1644-50.

31. Maurer MS, Spevack D, Burkhoff D, Kronzon I. Diastolic dysfunction: can it be diagnosed by Doppler echocardiography? J Am Coll Cardiol. 2004;44: 1543-9.

\section{Submit your next manuscript to BioMed Central and we will help you at every step:}

- We accept pre-submission inquiries

- Our selector tool helps you to find the most relevant journal

- We provide round the clock customer support

- Convenient online submission

- Thorough peer review

- Inclusion in PubMed and all major indexing services

- Maximum visibility for your research

Submit your manuscript at www.biomedcentral.com/submit
) Biomed Central 